\title{
Comparison of Micro-Shear Bond Strength of Resin Cement to Zirconia With Different Surface Treatments Using Universal Adhesive and Zirconia Primer
}

\author{
Baharan Ranjbar Omidi', Parnian Karimi Yeganeh', Sonia Oveisi², Negin Farahmandpour ${ }^{3}$, Fahimeh Nouri"* \\ 'Department of Operative Dentistry, School of Dentistry, Qazvin University of Medical Sciences, Qazvin, Iran \\ ${ }^{2}$ Department of Metabolic Research Center, Tehran University of Medical Sciences, Tehran, Iran \\ ${ }^{3}$ Restorative Dentistry Specialist, Hjørring, Denmark
}

\author{
*Correspondence to \\ Fahimeh Nouri, DMD, MS: \\ Assistant Professor, Department \\ of Operative Dentistry, School of \\ Dentistry Qazvin University of \\ Medical Sciences, Qazvin, Iran. \\ Tel: +989124799376; \\ Email: nouri.fahimeh@yahoo.com
}

Published online 28 July 2018

\begin{abstract}
Introduction: Increased demand for metal free fixed partial denture in recent years led to the developing of all ceramic material with excellent mechanical properties. One of the most popular all ceramic is zirconia which shows poor bonding properties. Recently, universal primer contains of silane and phosphate monomer for bond to zirconia have been introduced. The aim of this study is determination of the best method for bonding to zirconia based on the selection of the correct primer, suitable adhesive and best surface pretreatment.

Methods: In this in vitro experimental study 16 sintered-zirconia blocks prepared in dimension of $(18 \times 6 \times 2 \mathrm{~mm})$ by CAD/CAM technology. Sample cleansed by ultrasonic device contain of $96 \%$ ethanol in 6 minutes, after air-drying, based on surface treatment randomly divided into 4 groups which each group divided into 2 sub-groups based on the use of a primer or universal bond: (1) no treatment: (a) cement + zirconia primer, (b) cement + universal bond. (2) Alumina pretreatment: (a) cement + zirconia primer, (b) cement + universal bond. (3) Cojet sand pretreatment: (a) cement + zirconia primer, (b) cement + universal bond. (4) laser pretreatment (a) cement + zirconia primer, (b) cement + universal bond. Composite disc prepared with condensation of composite resin in Tygon tube with integral diminution of $0.7 \mathrm{~mm}$ which cured for 40 sconds. Universal bond or zirconia primmer apply on the surface of the zirconia samples then cemented to composite disks by Panavia F2 according manufacture instructions. Microshear bond strength determined with universal testing machine. Failure mode assessed under stereomicroscope. Selected sample based on surface treatment evaluated under SEM. Data were analyzed using one-way analysis of variance (ANOVA).

Results: Comparison of the 4 surface treatment groups revealed a significant difference and the highest bond belonged to Cojet and the lowest one to laser group.

Conclusion: It seems that Universal Adhesive can to be considered as an alternative to bond to zirconia but the Cojet method is still required.

Keywords: Universal bond; Zirconia; Micro-shear bond strength; Er: YAG laser.
\end{abstract}

\section{Introduction}

High demand for metal-free fixed partial dentures in the recent years led to the development of all-ceramic restorations with optimized mechanical properties. Zirconia restorations are the most commonly used among all-ceramic restorations due to unique mechanical properties such as flexural strength over $1000 \mathrm{MPa}$, chemical stability, and biocompatibility, as well as favorable optical properties that show excellent outcome in terms of clinical service and aesthetics. ${ }^{1}$
However, bond to zirconia has always been challenging. Zirconia has a polycrystalline structure. A reliable bond to zirconia increases marginal adaptation, prevents leakage and increases retention. Several techniques have been proposed to overcome the limits of bond to zirconia. The first solution was surface abrasion or surface roughening to create micromechanical retention and stabilize the bond to zirconia. In this method, air-borne alumina 50$100 \mu \mathrm{m}$ size particles are used to roughen and clean the zirconia surface. However, some studies have reported 
the formation of cracks and defects in zirconia following air abrasion by alumina particles in a way that the restoration becomes susceptible to fracture in function. ${ }^{2}$ The combination of alumina and silica particles is known as silica coating. It suggested enhanced micromechanical retention. In this method, a non-uniform silica layer is formed over the bonding surface; however, this layer shows variable effects on bond strength. ${ }^{3,4}$ Lasers have also been used to increase the bond to zirconia. Laser irradiation creates macroscopic and microscopic porosities on the ceramic surface, which raises the micromechanical retention of the resin cement and zirconia. However, it may also create cracks on the surface and lead to fracture of the restoration. ${ }^{5-8}$

The use of phosphate-ester primers is considered as another technique to increase bond strength to zirconia. These functional monomers create hydrogen bonds to the zirconia-resin interface; methacryloyloxyethyl dihydrogen phosphate (MDP)-containing primers are the most commonly used primers. Universal primers containing silane and phosphate monomers were recently introduced to the market as the last generation of adhesives for bonding to zirconia, which have been less commonly studied. ${ }^{9}$ The manufacturers claim that these agents can provide excellent bond to zirconia without any surface treatment.

Comprehensive studies comparing different combinations of zirconia surface treatments and zirconia primer or universal adhesives are scarce. ${ }^{8,10}$ Thus, this study aimed to assess the micro-shear bond strength of resin cement to zirconia with different surface treatments using Universal Adhesive and Z-Prime Plus to find the most efficient technique for clinical setting. The null hypothesis was no significant difference in bond strength of resin cement to zirconia with different surface treatment or the use of Z-Prime Plus primer or Universal Adhesive.

\section{Methods}

This in vitro experimental study was conducted on 120 ceramic blocks in 4 groups (depending on the type of surface treatment). Each group was randomly divided into 2 subgroups for bonding with Universal Adhesive or Z-Prime Plus primer. The sample size was calculated to be 14 samples in each subgroup according to a study by Amaral et $\mathrm{al}^{11}$ in which they considered $\alpha=0.05, \beta=0.8$ and $80 \%$ power of the study. To compensate for the possible dropouts, 15 samples were fabricated for each subgroup. Characteristics of the materials used in this study are presented in Table 1.

\section{Preparation of Zirconia Ceramic Samples}

Twenty zirconia blocks measuring $2 \times 6 \times 18 \mathrm{~mm}$ were fabricated using CAD/CAM technology. The blocks were sintered according to the manufacturer's instructions and cleaned in an ultrasonic bath containing $96 \%$ ethanol for 6 minutes.

Preparation of Z250 Composite Blocks

A2 shade of Z250 composite resin was incrementally applied to a silicone mold (Tygon tube Saint-Gobain, Paris, France) $0.7 \mathrm{~mm}$ in diameter and $2 \mathrm{~mm}$ in height on a glass slab and then condensed. Another glass slab was placed over it and each layer was light-cured for 40 seconds using a light-curing unit (Demetron, Kerr, Orange, CA, USA). The tube was separated from the composite and removed by a surgical blade. The fabricated composite cylinders were light cured for 40 seconds from all directions, then the samples were placed at $37^{\circ} \mathrm{C}$ for 24 hours to allow complete polymerization.

Surface Preparation of Ceramic Samples

Zirconia blocks were divided into 4 groups $(n=30)$ depending on the type of surface treatment as follows.

(1) No treatment (control group) $(\mathrm{n}=15)$.

(2) Sandblasting: Ceramic blocks were sandblasted by 50 $\mu \mathrm{m}$ alumina particles with 2.5 Psi pressure at $10 \mathrm{~mm}$ distance from the surface for 10 seconds. The blocks were then cleaned in an ultrasonic bath containing $96 \%$ ethanol for 6 minutes. Samples in this group were divided into 2 subgroups of (II A) Sandblasting plus Z-Prime Plus and (II B) Sandblasting plus Universal Adhesive $(n=15)$.

(3) Silica coating using Cojet Sand (3M, Blast Coating Agent, USA): Samples were sandblasted at 1-10 $\mathrm{mm}$ distance and 1-3 bar pressure perpendicular to the surface for 15 seconds. The excess coating material was cleaned by oil-free air. Samples in this group were divided into 2 subgroups of (III A) Cojet plus Z-Prime Plus and (III B) Cojet plus Universal Adhesive $(n=15)$.

(4) Er:YAG (erbium: yttrium-alumina garnet) laser: Zirconia blocks were subjected to Er:YAG laser (

Table 1. Characteristics and Manufacturers of the Materials Used in This Study

\begin{tabular}{lll}
\hline Material & Composition & Manufacturer \\
\hline ICE zirconia & Zirconium -oxide ceramic for CAD-CAM & Zirkonzahn, Italy \\
Panavia F 2.0 & MDP containing resin cement (self-etching dual cure) & Kuraray, Kurashiki, Japan \\
Cojet Sand & Blast coating agent & 3 M ESPE, St. Paul, MN, USA \\
Z-Prime Plus & Primer of zirconia & Bisco IL, United States \\
Future bond & Universal dental adhesive & Voco GmbH, Coxhaven, Germany \\
Z250 composite & Light-cure composite & 3M ESPE, St. Paul, MN, USA \\
\hline
\end{tabular}


Deka US20D, Italy) irradiation under water coolant adjusted at maximum air/water percentage of 10 $\mathrm{ml} / \mathrm{min}$, with a $1 \mathrm{~mm}$ diameter non-contact tip. The surface of the samples was treated (2 W output power, $10 \mathrm{~Hz}$ frequency, $2940 \mathrm{~nm}$ wavelength, 200 $\mathrm{mJ}$ pulse energy, $18.51 \mathrm{~J} / \mathrm{cm}^{2}$ total energy density) from a distance of $10 \mathrm{~mm}$ perpendicular to the surface and an exposure time of 10 seconds. In fact, at lower distance, the possibility of zirconia cracks was enhanced, and eventually, the bond strengths would be declined. The blocks were cleaned in an ultrasonic bath containing 96\% ethanol for 6 minutes. This group was divided into 2 subgroups of (IV A) Laser plus Z-Prime Plus and (IV B) Laser plus Universal Adhesive. To assimilate samples preparation, all the specimens were prepared at the same distance.

\section{Assessment of Treated Surfaces Under SEM \\ One sample of each group was evaluated by SEM (AIS2100 SeronTechnologgies, South Korea). The samples were sputter coated with gold and evaluated on $\times 5$ and $\times 10$ magnifications.}

Bonding the Composite Blocks to Zirconia Surfaces Using Z-Prime Plus and Panavia Cemen

Z-Prime Plus was applied uniformly on the zirconia ceramic surfaces to cover the entire surface. The surfaces were gently dried with air spray for 2-5 seconds. Equal amounts of Panavia F2 pastes were mixed on a mixing pad for 20 seconds according to the manufacturer's instructions. The cement was applied to the surface of the ceramic blocks, and composite blocks were placed over it. A thirty MPa load was applied by a gage to each composite block to obtain equal cement thickness in all samples. Each block was light cured by an LED light curing unit (Demetron, Kerr, Orange, CA, USA) for 10 seconds and the excess cement was removed. Then, final polymerization was achieved by 30 seconds of light curing. Samples were stored in deionized water at $37^{\circ} \mathrm{C}$ for 24 hours.

Bonding the Composite Blocks to Zirconia Surfaces Using Universal Adhesive and Panavia Cement

Universal Adhesive was mixed and applied to the ceramic surfaces for 20 seconds using a micro-brush followed by air-drying for 5 seconds with air spray to evaporate the solvent. With the Panavia cement, composite blocks bonded to the zirconia surfaces as described earlier.

\section{Micro-Shear Bond Strength Testing}

All samples were subjected to 2500 thermal cycles between $5-55^{\circ} \mathrm{C}$ with a dwell time of 30 seconds and transfer time of 15 seconds.

Each sample was glued to the universal testing machine (Zwick Roell, Ulm, Germany) using cyanoacrylate glue. Micro-shear bond strength was measured using wire and loop method. A thin wire with $0.2 \mathrm{~mm}$ diameter was tied to each composite cylinder, making contact with the lower half of the cylinder. On the opposing side, the wire was tied to bars designed for this purpose. The connection between the cylinder, wire, and rods was positioned in the same line to ensure proper shear load distribution at the interface. Prior to load application, wire and loop should be as close as possible to the interface. Next, shear load was applied at a crosshead speed of $0.5 \mathrm{~mm} / \mathrm{min}$ until failure occurred. The load at failure was recorded in Newton (displayed on the monitor). Micro-shear bond strength in MPa was calculated by dividing the load in $\mathrm{N}$ by the surface area of the composite cylinder (contact surface area with $0.7 \mathrm{~mm}$ diameter).

Determination of the Mode of failure

Mode of failure was determined by observation of fracture on surfaces under a stereomicroscope (YS100, Nikon, Sweden) at $\times 40$ magnification. Mode of failure was determined as adhesive (at the resin cement-ceramic interface), cohesive (within the composite) or mixed (a combination of both adhesive and cohesive).

\section{Statistical Analyses}

The data were analyzed using SPSS version 19. Normal distribution of data was assessed by Kolmogorov-Smirnov test, which showed that the data had normal distribution. Thus, one-way Analysis of variance (ANOVA) was applied for comparison of the groups. To compare the Universal Adhesive and Z-Prime Plus subgroups, independent $t$ test was used. $P<0.05$ was considered statistically significant.

\section{Results}

\section{Results of Bond Strength Testing}

During thermocycling, one sample of the no treatment group and 14 samples in the laser group were lost due to fracture. The mean and standard deviation of shear bond strength in the remaining samples are presented in Table 2.

Comparison of Universal Adhesive and Z-Prime Plus subgroups by independent $t$ test showed significant differences in all groups, bond strength in the Universal Adhesive subgroup was higher than that of the Z-Prime Plus subgroup $(P<0.05)$.

One-way ANOVA was applied to compare the $4 \mathrm{Z}$-Prime Plus subgroups and found a significant difference among them $(P<0.05)$. Pairwise comparison by Bonferroni adjustment post hoc test revealed that the lowest bond strength belonged to laser and Z-Prime Plus and the highest to Cojet plus Z-Prime Plus subgroup.

Pairwise comparisons revealed significant differences between the subgroups except for the no treatment and sandblasted groups, which were not significantly different. Comparison of the 4 surface treatment groups revealed a significant difference and the highest bond belonged to Cojet and the lowest to laser group. 
Table 2. Comparison of Universal Adhesive and Z-Prime Plus Subgroups With Different Surface Treatments

\begin{tabular}{|c|c|c|c|c|c|}
\hline Study Groups & & Number of Samples & Lost Samples & Mean Bond Strength & Standard Deviation \\
\hline \multirow{2}{*}{ No treatment } & Z-Prime Plus & 14 & 1 & 6.49 & 3.12 \\
\hline & Universal Adhesive & 15 & 0 & 23.38 & 3.43 \\
\hline \multirow{2}{*}{ Sandblast } & Z-Prime Plus & 15 & 0 & 19.16 & 3.53 \\
\hline & Universal Adhesive & 15 & 0 & 25.70 & 2.01 \\
\hline \multirow{2}{*}{ Cojet } & Z-Prime Plus & 15 & 0 & 22.21 & 2.69 \\
\hline & Universal Adhesive & 15 & 0 & 32.54 & 4.28 \\
\hline \multirow{2}{*}{ Laser } & Z-Prime Plus & 6 & 9 & 1.59 & 1.19 \\
\hline & Universal Adhesive & 10 & 5 & 5.40 & 2.71 \\
\hline
\end{tabular}

\section{Mode of Failure}

In no treatment, laser and sandblast Z-Prime Plus subgroup, all fractures were adhesive while in the no treatment, laser and sandblast Universal Adhesive subgroups, most fractures were mixed. In the laser group, all fractures in both subgroups were adhesive.

\section{Results of SEM Analysis}

Evaluation of zirconia surfaces under SEM revealed irregular surface roughness of sandblasted surfaces. Defects and cracks exist on laser irradiated surfaces. Silicacoated samples showed more uniform surface roughness compared to sandblasted surfaces (Figure 1A-D).

\section{Discussion}

High-strength zirconia ceramics are extensively used in dentistry. Resin cement is recommended for these restorations to increase marginal adaptation, retention and fracture resistance of zirconia ceramics. ${ }^{12}$ The clinical success of zirconia restorations highly depends on their cementation process. To obtain a stable bond, different surface preparation methods have been proposed. ${ }^{12}$ High-strength ceramics do not contain a glass phase; thus, conventional surface preparations have no effect on them. ${ }^{7}$ Studies are ongoing to find the most efficient
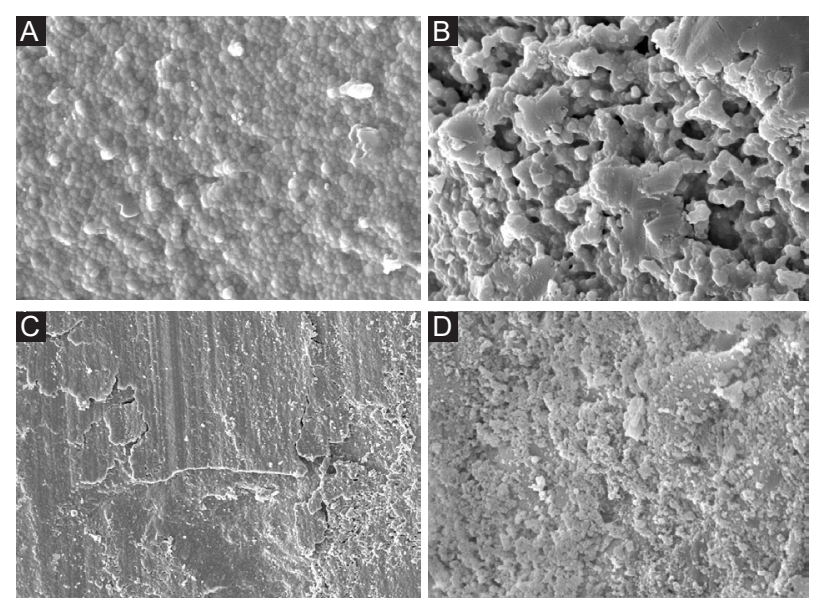

Figure 1. SEM micrograph of (A) the surface with no treatment, (B) the sandblasted surface, (C) the laser irradiated surface, and (D) the Cojet treated surface at $\times 10$ magnification. surface treatment for zirconia ceramics.

In vitro mechanical tests on samples allows recognition of materials and methods with the greatest potential for use in the clinical setting. ${ }^{13}$ Shear, tensile, micro-shear, and micro-tensile tests have been suggested for assessment of bond strength of ceramic and resin cement. ${ }^{14}$

In the current study, ceramic blocks were bonded to composite blocks instead of teeth to standardize the samples and prevent possible complications associated with the use of tooth structure. ${ }^{15}$ Also, Panavia resin cement containing phosphate monomers (10-MDP) was used since it is the most commonly used and efficient cement for all ceramic restorations. ${ }^{16}$ High stability of the bond provided by this cement is due to the direct bond of esterase groups of MDP to metal oxides. ${ }^{17}$

Use of silane to enhance the bond strength of resin to ceramic has been previously evaluated. Silane molecules have dual reactions, one of which reacts with water to form three silanol $(-\mathrm{Si}-\mathrm{OH})$ groups that react with silica on the ceramic surface and create siloxane ( $\mathrm{Si}-\mathrm{O}-\mathrm{Si}-\mathrm{O}$ ) molecules. The methacrylate end of the silane molecule reacts with the methacrylate groups in resin cement and form chemical bonds. However, silane is used for ceramics that contain a glass phase. For high-strength ceramics without a glass phase, silica coating systems are used, which form a silica layer on the ceramic surface and enable the use of silane on the surface of high-strength ceramics. ${ }^{16}$

Since conventional methods cannot be used for zirconia surface preparation, other methods such as using primers with chemical affinity for metal oxides were suggested. These primers include 10-MDP, 4-methacryloyloxyethyl trimellitate anhydride(4-META) and 2-methacryloyloxyethyl ethyl phosphate, which can react with oxide groups in zirconia ceramics. ${ }^{18}$

In our study, sandblasted samples showed higher bond strength than control samples. Universal Adhesive sandblasted samples showed higher bond strength than Z-Prime Plus primer sandblasted samples. Nagayassue et $\mathrm{al}^{19}$ and Wolfart et $\mathrm{al}^{20}$ evaluated the effect of different surface treatments on resin cement bond to zirconia and alumina ceramics, in which $50 \mu \mathrm{m}$ alumina sandblasting particles increased the bond strength. Conversely, 
Amaral et $\mathrm{al}^{11}$ indicated that the use of $110 \mu \mathrm{m}$ alumina sandblasting particles was not effective in increasing the micro-tensile bond strength of zirconia to resin cement. Tsuo et $\mathrm{al}^{21}$ concluded that the large size of alumina particles created deep grooves on the ceramic surface and decreased the wettability and contact angle of ceramic with silane and resin cement. It has been reported that sandblasting with 30-50 $\mu \mathrm{m}$ alumina particles increases the bond strength of zirconia to resin cement. ${ }^{22}$ In the current study, sandblasting with $50 \mu \mathrm{m}$ alumina particles was performed. However, mechanical retention is not sufficient for efficient bonding. For this reason, several adhesive systems were introduced to form a chemical bond to zirconia. Z-Prime Plus is a primer containing HEMA and ethanol. It does not contain water or a high molecular weight monomer such as Bis-GMA. Universal Adhesive is another recently introduced adhesive for enhancing bond of resin cement to zirconia.

Our study showed a significant increase in bonding of resin cement to zirconia when Cojet surface treatment was used. This finding was in accordance with the results of Amaral et al. ${ }^{11}$ They reported the highest tensile bond strength in sandblasted groups with Rocatec and Siljet systems. Kiyan et al in 2007 also reported that the use of silica coating was the most effective method of surface preparation for alumina ceramics. Although the size of the particles in their study was different from the current study. ${ }^{23}$ Sandblasting with Cojet forms a silica layer over the zirconia surface, which forms a chemical bond between the primer or Universal Adhesive and the zirconia surface..$^{24}$

Adhesion is influenced by mechanical, chemical and thermal factors. Thermocycling was performed in the current study to simulate thermal changes in the clinical oral environment. However, chemical and mechanical factors were not simulated, which counts as a limitation of the current study. Samples were subjected to 2500 thermal cycles, which resulted in pretest fracture of samples with low bond strength in the laser group. Evidence shows that thermocycling decreases the resin cement bond to zirconia. ${ }^{25-27}$ Hydrolytic degradation, water sorption, and thermal alterations all decrease the bond strength at the interface. ${ }^{28}$

With the advances in laser technology, laser irradiation has also been suggested for zirconia surface preparation and enhancing the cement bond to zirconia. Er:YAG laser has shown promising results for dental applications compared to other lasers and has less destructive effects. ${ }^{6}$ Thus, Er: YAG laser was used in this study with $200 \mathrm{~mJ}$ power under water coolant (to prevent tension due to over-heating). As reported earlier, 14 out of 30 samples in the laser group deboned prior to testing, which shows that Er:YAG laser with the applied parameters cannot provide a durable bond between zirconia and resin cement. It appears that inappropriate power output created cracks on the zirconia surface and the weak bond between these cracks and the underlying layers caused debonding. The SEM micrographs clearly show these microcracks on the zirconia surface. Erdem et al demonstrated the same result as in the current study for shear bond strength of surfaces treated with Er:YAG laser. Laser parameters were similar to those used in our study. There was no significant difference between no treatment surfaces and laser application. ${ }^{10}$ They referred the presence of cracks seen on SEM micrographs as a reason of lower bond strength. Also, our findings confirm Foxton et al results, who used sandblasting and Er: YAG laser to enhance the bond of dual-cure resin cement to zirconia and Procera ceramics. ${ }^{5}$

In all groups in our study, Universal Adhesive subgroups showed higher bond strength than Z-Prime Plus primer subgroups. Previous in vitro studies have also confirmed our findings in that Universal Adhesives provide suitable bond even to dentin. ${ }^{29,30}$ High bond strength reported for Universal Adhesive is due to the presence of an MDP monomer, which has a strong affinity for dentin, enamel, and metals. Moreover, it also forms a chemical bond to zirconia (Zr-O-P). It has been documented that adhesives containing MDP monomer create an interface resistant to biodegradation due to forming chemical bonds with the residual hydroxyapatite crystals when used with selfetch adhesives on dentin. Some new formulations contain silane to enhance adhesion to ceramic surfaces. ${ }^{27}$

Universal Adhesive can perfectly enhance the bond strength to zirconia without changing its microscopic structure. The superiority of Universal Adhesive is that it can be used for a wide range of substrates and eliminates the risk of inappropriate preparation of bonded surfaces. Also, simple application increases acceptance for use in clinical procedures. Thus, it may serve as a suitable alternative to primers. Moreover, Universal Adhesive can be used for repair of broken substrates. The current study results showed that the application of Universal Adhesive increased the bond strength even in the absence of surface treatment.

In the current study, mixed failures had the highest frequency in all Universal subgroups (except for the laser plus Universal Adhesive subgroup), which indicates higher bond strength provided by Universal Adhesive compared to Z-Prime Plus primer. In the laser group, all fractures were adhesive due to low bond strength. Modes of failure in the study groups were in accordance with the findings of bond strength tests.

The durability of the ceramic bond to resin cement provided by the use of Universal Adhesive must be evaluated in long-term studies. Also, the efficacy of Universal Adhesive for repair of ceramic restorations with composite resin can be an interesting topic for future studies.

\section{Conclusion}

Within the limitations of this in vitro study, the following 
results were obtained:

1. Silica coating, irrespective of the use of primer or Universal Adhesive, provided significantly higher micro-shear bond strength values than other methods (no treatment, sandblasting, laser).

2. Silica coating Universal Adhesive yielded the highest micro-shear bond strength.

3. Bond strength values of sandblasting were superior to Er:YAG laser and no surface treatment.

4. Er: YAG laser is not suitable for enhancing the bond of zirconia to resin cement.

5. Application of Universal Adhesive even in absence of surface treatment increases the bond strength to zirconia.

\section{Ethical Considerations}

The proposal of the study was approved by the Ethics Committee, deputy of research, Qazvin University of Medical Sciences (\#1394.63).

\section{Conflict of Interests}

This research was supported by research \& development department of Qazvin Dentistry School.

\section{Acknowledgment}

The authors would like to thank you the "Dental Caries Prevention Research Center, Qazvin University of Medical Sciences, Qazvin, Iran” for their supports.

\section{References}

1. Aboushelib MN, de Jager N, Kleverlaan CJ, Feilzer AJ. Microtensile bond strength of different components of core veneered all-ceramic restorations. Dent Mater. 2005;21(10):984-991. doi:10.1016/j.dental.2005.03.013

2. Guess PC, Zhang Y, Kim JW, Rekow ED, Thompson VP. Damage and reliability of Y-TZP after cementation surface treatment. J Dent Res. 2010;89(6):592-596. doi:10.1177/0022034510363253

3. Heikkinen TT, Lassila LV, Matinlinna JP, Vallittu PK. Effect of operating air pressure on tribochemical silicacoating. Acta Odontol Scand. 2007;65(4):241-248. doi:10.1080/00016350701459753

4. Ozcan M. The use of chairside silica coating for different dental applications: a clinical report. J Prosthet Dent. 2002;87(5):469-472.

5. Foxton RM, Cavalcanti AN, Nakajima M, et al. Durability of resin cement bond to aluminium oxide and zirconia ceramics after air abrasion and laser treatment. $J$ Prosthodont. 2011;20(2):84-92. doi:10.1111/j.1532849X.2010.00678.x

6. Akyil MS, Uzun IH, Bayindir F. Bond strength of resin cement to yttrium-stabilized tetragonal zirconia ceramic treated with air abrasion, silica coating, and laser irradiation. Photomed Laser Surg. 2010;28(6):801-808. doi:10.1089/pho.2009.2697

7. Cavalcanti AN, Foxton RM, Watson TF, Oliveira MT, Giannini M, Marchi GM. Bond strength of resin cements to a zirconia ceramic with different surface treatments.
Oper Dent. 2009;34(3):280-287. doi:10.2341/08-80

8. Spohr AM, Borges GA, Junior LH, Mota EG, Oshima HM. Surface modification of In-Ceram Zirconia ceramic by Nd:YAG laser, Rocatec system, or aluminum oxide sandblasting and its bond strength to a resin cement. Photomed Laser Surg. 2008;26(3):203-208. doi:10.1089/ pho.2007.2130

9. Attia A, Lehmann F, Kern M. Influence of surface conditioning and cleaning methods on resin bonding to zirconia ceramic. Dent Mater. 2011;27(3):207-213. doi:10.1016/j.dental.2010.10.004

10. Erdem A, Akar GC, Erdem A, Kose T. Effects of different surface treatments on bond strength between resin cements and zirconia ceramics. Oper Dent. 2014;39(3):E118-127. doi:10.2341/12-420-1

11. Amaral R, Ozcan M, Bottino MA, Valandro LF. Microtensile bond strength of a resin cement to glass infiltrated zirconia-reinforced ceramic: the effect of surface conditioning. Dent Mater. 2006;22(3):283-290. doi:10.1016/j.dental.2005.04.021

12. Atsu SS, Kilicarslan MA, Kucukesmen HC, Aka PS. Effect of zirconium-oxide ceramic surface treatments on the bond strength to adhesive resin. J Prosthet Dent. 2006;95(6):430436. doi:10.1016/j.prosdent.2006.03.016

13. Ural C, KalyoncuoGlu E, Balkaya V. The effect of different power outputs of carbon dioxide laser on bonding between zirconia ceramic surface and resin cement. Acta Odontol Scand. 2012;70(6):541-546. doi:10.3109/00016357.2011.60 0718

14. Valandro LF, Ozcan M, Amaral R, Vanderlei A, Bottino MA. Effect of testing methods on the bond strength of resin to zirconia-alumina ceramic: microtensile versus shear test. Dent Mater J. 2008;27(6):849-855.

15. Casucci A, Monticelli F, Goracci C, et al. Effect of surface pre-treatments on the zirconia ceramic-resin cement microtensile bond strength. Dent Mater. 2011;27(10):10241030. doi:10.1016/j.dental.2011.07.002

16. Della Bona A. Bonding to ceramics: scientific evidences for clinical dentistry. Sao Paulo: Artes Médicas; 2009.

17. Sun R, Suansuwan N, Kilpatrick N, Swain M. Characterisation of tribochemically assisted bonding of composite resin to porcelain and metal. J Dent. 2000;28(6):441-445.

18. Attia A, Kern M. Long-term resin bonding to zirconia ceramic with a new universal primer. J Prosthet Dent. 2011;106(5):319-327. doi:10.1016/s0022-3913(11)60137-6

19. Nagayassu MP, Shintome LK, Uemura ES, Araujo JE. Effect of surface treatment on the shear bond strength of a resinbased cement to porcelain. Braz Dent J. 2006;17(4):290295.

20. Wolfart M, Lehmann F, Wolfart S, Kern M. Durability of the resin bond strength to zirconia ceramic after using different surface conditioning methods. Dent Mater. 2007;23(1):45-50. doi:10.1016/j.dental.2005.11.040

21. Tsuo Y, Yoshida K, Atsuta M. Effects of alumina-blasting and adhesive primers on bonding between resin luting agent and zirconia ceramics. Dent Mater J. 2006;25(4):669674.

22. Magne P, Paranhos MP, Burnett LH, Jr. New zirconia primer improves bond strength of resin-based cements. Dent Mater. 2010;26(4):345-352. doi:10.1016/j.dental.2009.12.005 
23. Kiyan VH, Saraceni $\mathrm{CH}$, da Silveira BL, Aranha AC, Eduardo Cda P. The influence of internal surface treatments on tensile bond strength for 2 ceramic systems. Oper Dent. 2007;32(5):457-465. doi:10.2341/06-131

24. Matinlinna JP, Lassila LV, Vallittu PK. The effect of five silane coupling agents on the bond strength of a luting cement to a silica-coated titanium. Dent Mater. 2007;23(9):11731180. doi:10.1016/j.dental.2006.06.052

25. Koizumi H, Nakayama D, Komine F, Blatz MB, Matsumura $\mathrm{H}$. Bonding of resin-based luting cements to zirconia with and without the use of ceramic priming agents. $J$ Adhes Dent. 2012;14(4):385-392. doi:10.3290/j.jad.a22711

26. Lung CY, Botelho MG, Heinonen M, Matinlinna JP. Resin zirconia bonding promotion with some novel coupling agents. Dent Mater. 2012;28(8):863-872. doi:10.1016/j. dental.2012.04.023

27. Azimian F, Klosa K, Kern M. Evaluation of a new universal primer for ceramics and alloys. J Adhes Dent. 2012;14(3):275-282. doi:10.3290/j.jad.a22193

28. Matinlinna JP, Lassila LV. Experimental Novel Silane System in Adhesion Promotion between Dental Resin and Pretreated Titanium. Part II: Effect of Long-Term Water Storage. Silicon. 2010;2(2):79-85. doi:10.1007/s12633-0109039-6

29. Yoshida Y, Yoshihara K, Nagaoka N, et al. Self-assembled Nano-layering at the Adhesive interface. J Dent Res. 2012;91(4):376-381. doi:10.1177/0022034512437375

30. Perdigao J, Sezinando A, Monteiro PC. Laboratory bonding ability of a multi-purpose dentin adhesive. Am J Dent. 2012;25(3):153-158. 